\title{
APRENDIZAJE COLABORATIVO SOPORTADO POR COMPUTADOR (CSCL): SU ESTADO ACTUAL
}

\author{
Elsa Piedad Cabrera Murcia \\ Doctorando en Psicología, Pontificia Universidad Católica, Chile
}

\section{INTRODUCCIÓN}

En los últimos años, en Latinoamérica, se ha despertado un gran interés por el diseño de herramientas instruccionales educativas que potencien el desarrollo de habilidades y competencias en los estudiantes, pero que a su vez permitan el surgimiento de otras experiencias y dinámicas de trabajo dentro del aula. Dicho interés ha iniciado tempranamente en países como Estados Unidos, Inglaterra y Australia, los cuales cuentan en la actualidad con experiencias educativas que reflejan los beneficios que se alcanzan al trabajar con computadores portátiles dentro del aula.

Means (2002) afirma que veinticinco años de investigación con computadores fijos, han mostrado que la tecnología puede convertirse en una herramienta que, bien usada, puede impactar positivamente el proceso de enseñanza aprendizaje. Durante el 2001-2002 el Center for Technology in Learning (SRI's) inició investigaciones implementando dentro del aula el trabajo con computadores portátiles inalámbricos. Los resultados muestran que dicha herramienta puede ofrecer beneficios únicos tanto para profesores como para los estudiantes (Means, 2002). El $72 \%$ de los profesores afirman que el uso de las Palm (en este texto las llamaremos ADP) contribuyen positivamente al aprendizaje de los estudiantes y $84 \%$ de ellos, consideran que hacer uso de esta metodología permite mejorar la calidad de las actividades de aprendizaje implementadas (Tatar, Roschelle, Vahey \& Penuel, 2003).

También se ha encontrado que el trabajo con las ADP son efectivos en situaciones de aprendizaje activo, en donde los estudiantes responden preguntas, buscan, analizan la información y la elaboran en un producto final. Así mismo se ha encontrado que esta herramienta enriquece los ambientes en donde se construyen aprendizajes en forma colaborativa, es decir, cuando los estudiantes trabajan en conjunto, cara a cara con sus pares (Torgrude, 2001).

Partiendo entonces de la actual relevancia que posee el trabajo colaborativo haciendo uso de computadores portátiles inalámbricos como herramienta que media el aprendizaje de los estudiantes, se hace necesario recopilar el estado actual de esta nueva metodología para que desde allí, podamos contextualizar dicho trabajo a nuestra realidad Latinoamericana. En este sentido, dicha revisión intentará clarificar las siguientes preguntas:

- ¿Qué tipo de investigaciones se han realizado con el aprendizaje colaborativo soportado con computador?

- ¿Cuáles han sido los beneficios y desventajas obtenidas con su aplicación, en que áreas y poblaciones?

- ¿Cuál ha sido el foco de atención de los aprendizajes colaborativos soportados por computador en los últimos tiempos?

- ¿ ¿Cuáles pueden ser las posibles direcciones de investigación a seguir? 
En principio en este artículo se explora el marco conceptual en el que se enmarca el uso de la tecnología, como metodología que favorece el proceso de enseñanzaaprendizaje. Posteriormente se presenta un conjunto de investigaciones que reportan las fortalezas y debilidades que se obtienen al hacer uso de esta metodología, y en específico, el uso de computadores inalámbricos portátiles dentro del aula (ADP). Asimismo, se presentan las contradicciones o inconsistencias que se encuentran en los estudios revisados, para finalmente explicitar las posibles líneas de investigación que permitirán fundamentar el trabajo con dicha herramienta dentro del aula.

\section{MARCO CONCEPTUAL}

El trabajo en conjunto entre pares, entre novatos y expertos o en comunidades de aprendices establecidas, cuenta con una gran cantidad de evidencia empírica que fundamenta que la interacción social trae beneficios al aprendizaje (Rogoff, 1993; Vigotsky, 1996). En ocasiones a este tipo de trabajo se le ha llamado cooperativo o colaborativo según las características de organización, o los roles que se establecen dentro de los grupos. Es así como en la literatura se puede encontrar un sinnúmero de definiciones que los usan desde sinónimos hasta aquellos que los caracterizan como dos conceptos antónimos. Por tanto se hace necesario en el desarrollo de esta síntesis, describir el marco de lo que en adelante se entenderá por aprendizaje colaborativo.

Muhlenbrock (1999) considera que el aprendizaje colaborativo es el compromiso mutuo establecido entre un grupo de personas, que se agrupan en un esfuerzo coordinado para dar respuesta a una tarea. Para él, este tipo de organización permite entender los procesos que se gestan al trabajar entre pares. Dillenbourg (1999) entre tanto, afirma que la clave para entender el aprendizaje colaborativo es reconocer las relaciones que se establecen entre la situación que se plantea, las interacciones que emergen y en consecuencia, los procesos y efectos que se generan en ella. Estos cuatro elementos que se describen a continuación se constituyen en los elementos clave que deben tenerse en cuenta al momento de evaluar un contexto de aprendizaje colaborativo (Dillenbourg, 1999):

- la situación, establecida a partir del grado de simetría de las acciones, el conocimiento y el estatus de los participantes para dar resolución a la tarea en forma conjunta.

- las interacciones, enmarcadas dentro de la situación colaborativa que se ha establecido. Éstas pueden ser interactivas, sincrónicas y negociables. Dichas interacciones influyen en los procesos cognitivos de cada uno de los participantes.

- Los mecanismos de aprendizaje, obtenidos a partir de la interacción entre pares. Éstos pueden ser aquellos que operan en el caso de la cognición individual, como aquellos que operan a nivel grupal como la apropiación, el mutuo modelamiento y la internalización.

- Los efectos del aprendizaje colaborativo, generalmente medidos a partir de un pretest o postest con los cuales se pretende obtener una medición de las ganancias que han obtenido los estudiantes.

En definitiva, la clave para entender los procesos que se dan al interior del aprendizaje colaborativo son las relaciones bidireccionales que se establecen entre estos cuatro elementos. En principio "... la situación genera patrones de interacción, estas interacciones gatillan mecanismos cognitivos, que a su vez generan efectos cognitivos" (p.18). En la modalidad de aprendizaje colaborativo haciendo uso de 
herramientas tecnológicas (Computer-Supported Collaborative Learning - CSCL) se presentan los mismos elementos, pero la dinámica organizacional varía por el uso de una herramienta (computador) que media el aprendizaje colaborativo.

Roschelle, Pea, Gordin \& Means (2001) reportan en sus investigaciones que la enseñanza mediada por el computador lidera un gran cambio de la escuela, los profesores, los estudiantes y no en menor grado de importancia, del currículo. En otras palabras, la mediación de ésta herramienta no solo origina cambios en la forma como se estructura la organización escolar, sino que también reporta beneficios en el aprendizaje de los estudiantes. Estos últimos pueden resumirse en a) habilidades de alto orden de pensamiento crítico (Roschelle et al, 2001), b) autonomía en el aprendizaje y colaboraciones más efectivas (Tatar et al, 2003) y, c) habilidades sociales personales y de grupo (Lucero, 2003).

Dicha metodología de enseñanza tiene como cimientos dos perspectivas del aprendizaje que se complementan: la constructivista y la teoría de la actividad (TA), que permiten representar las actividades de grupos donde la tecnología juega un rol como mediador en el aprendizaje (Barros, Mizoguchi \& Verdejo, 2001). Desde la mirada constructivista el CSCL ve al estudiante como un agente activo, constructor de su proceso de aprendizaje, una persona que posee y genera conocimiento. Aunque esta perspectiva puede integrarse en las aulas con o sin soporte computacional, Roschelle et al (2001, p.6) afirman que las características del soporte tecnológico la hacen una "herramienta particularmente útil para este tipo de aprendizaje".

Por otro lado desde la TA, lo relevante es que la actividad interna en el individuo emerge desde la actividad externa que se origina dentro de su ambiente cultural. Partiendo de esta premisa el aprendizaje es visto como un proceso complejo, en donde las cogniciones individuales son definidas por las relaciones establecidas entre la situación, las herramientas y las formas de interacción que se dan dentro de dichas situaciones (Gifford \& Enyedy, 1999). En otras palabras diferentes elementos hacen parte activa del proceso de aprendizaje, a saber: la comunidad que a su vez involucra normas sociales que gobiernan la situación, la división de la labor, los roles que se juegan al interior del grupo, las herramientas para trabajar, los sujetos de la actividad, el objeto de la actividad y finalmente el resultado producido, en este caso por el grupo (Cole, 1999).

Dicha teoría como marco metodológico del CSCL permite entender la actividad que se genera en esta forma de trabajo, como interdependiente de los otros elementos que se requieren para que exista actividad. Tanto la comunidad (los otros) como las herramientas (computador) son fuentes que el estudiante (agente) coordina durante el desarrollo de la actividad (objetivo). Su acción y la de los otros, se ve modificada por las reglas y los roles que emergen en la interacción. Finalmente como las relaciones que se establecen son bidireccionales, el resultado pueda transformarse en una nueva actividad o en un nuevo artefacto. Nardi, (1996 citado en Roschelle, 2002) clarifica lo explicitado anteriormente haciendo uso de la siguiente representación gráfica:

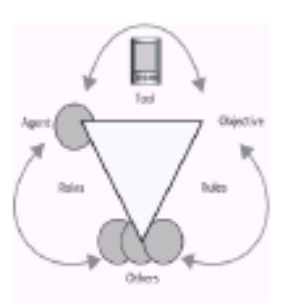


Gráfico 1: Teoría de la actividad como marco del CSCL

(Tomado de Roschelle, 2002)

Al hablar de CSCL es importante hacer la diferenciación entre trabajo colaborativo y aprendizaje colaborativo, ya que pueden llevar a confusiones al momento de analizar el tema que nos ocupa, a pesar de que ambas comparten las interacciones como base del éxito grupal. El primero o CSCW, es usado a nivel organizacional en donde la división de las labores está definida de antemano y le facilitan al individuo alcanzar aprendizajes relacionados con los objetivos de la organización (Lucero, 2003). Por el contrario el CSCL se da en contextos de aprendizaje escolar donde el desarrollo personal y grupal se constituye en el vector de su funcionamiento, y donde la división de las labores no está predeterminada.

Por lo general los ambientes CSCL involucran estudiantes distribuidos en grupos que trabajan juntos sobre un problema o proyecto común. En estos espacios colaborativos se presentan múltiples agentes que deben coordinar múltiples representaciones superficiales y conceptuales. Las primeras guardan relación con las diferentes formas de representación de un contenido y las segundas, hacen referencia a diferencias en sistemas conceptuales o modos de aproximación a un contenido en particular (Bruggen, Kirschner \& Jochems, 2002). Por ejemplo en física, los estudiantes deben coordinar diferentes formas de experimentar con la presión ejercida por los líquidos (representaciones superficiales) pero a su vez, deben esclarecer los diferentes conceptos que en estas representaciones se manejan como la densidad, la altura y aceleración de gravedad donde se encuentra el líquido (representaciones conceptuales).

\section{UNA MIRADA AL CSCL}

En esta sección se pretende dar respuesta a dos aspectos de interés: la metodología que ha sido usada para la evaluación de ambientes CSCL, las variaciones que se han dado en algunas aplicaciones tecnológicas fijas y portátiles, así como los resultados que se han alcanzado en especial con los CSCL inalámbricos dentro del contexto escolar.

a) Metodología

Durante los últimos años la metodología que guía las evaluaciones en estos ambientes se ha caracterizado por ser de corte cuantitativo y cualitativo. En éstas se trata de obtener cuáles son los resultados que alcanzan los estudiantes al trabajar con CSCL y las percepciones que tanto profesores como estudiantes tienen del manejo de computadores en el trabajo académico.

Dicha revisión muestra como se debe hacer un mayor acercamiento al estudio de CSCL a partir de variadas técnicas de corte cualitativo, que permitan recoger la información más cercana y real de los actores que participan en forma directa en el trabajo escolar. Asimismo se identifica que es un campo fértil de investigación, pues el CSCL se constituye en un paradigma relativamente nuevo en donde se requiere dar un mayor sustento a los hallazgos que se han alcanzado hasta el momento.

b) Herramientas tecnológicas fijas y portátiles en ambientes CSCL

Veerman, Andriessen \& Kanselaar (1999) reportaron tres estudios con estudiantes universitarios que trabajaban colaborativamente sobre tareas de discusión en ambientes electrónicos. A partir de un análisis de conglomerados de las discusiones emitidas por los participantes, encontraron que este tipo de 
trabajo favorece el desarrollo de discusiones constructivas. Es decir, argumentaciones construidas a partir de la adición, explicación, evaluación o síntesis que los estudiantes llevan a cabo cuando trabajan en grupo. Por ejemplo con Belvédère; una red que permite el trabajo individual y grupal para la construcción de diagramas argumentativos; se encontró que un $21 \%$ de las intervenciones tienen la función de verificar, un $10 \%$ de intercambiar y un $19 \%$ de contraargumentar. Esto estaría indicando que dicha herramienta estimula más a los estudiantes a revisar y comparar con mayor frecuencia la información con sus pares generando actividades constructivas, así como una tendencia a focalizarse en el uso y significado de los conceptos (Veerman, 2000).

Otros estudios son realizados en ambientes de aprendizaje colaborativo bas ados en la web para grupos que interactúan vía internet. Lea, Rogers \& Postmes (2002) afirman que esta modalidad permite el trabajo de estudiantes que no están cercanos geográficamente y que por esta misma razón los resultados que se obtienen pueden ser más ricos que los obtenidos en colaboraciones cara a cara. Haslam (2000, citado en Lea et al 2002) argumenta que una de las dificultades en estos ambientes cara a cara es justamente la baja congruencia entre la autodefinición de las personas y las características del ambiente en que se plantea la tarea.

De igual manera otras evidencias empíricas confirman dichos hallazgos. Por ejemplo Lê (2002) reporta que en su estudio con 180 estudiantes de la facultad de educación de la Universidad de Tasmania, la web fue usada por ellos como una herramienta que les permitía crear espacios de aprendizaje colaborativo. El $78 \%$ de los estudiantes de este estudio explicitaron la importancia de este trabajo para su posterior presentación escrita y el $21 \%$ la contribución positiva al desarrollo de su presentación oral.

Dentro de la literatura revisada se presentan también investigaciones de aprendizaje colaborativo soportadas con computadores portátiles (laptop), los cuales son considerados como herramientas que mejoran el proceso de enseñanza aprendizaje. Un estudio experimental se llevó a cabo a partir de dos condiciones: trabajo de estudiantes con y sin computador portátil (24 y 21 estudiantes respectivamente), con el fin de evidenciar los beneficios que trae al aula el trabajo con dicha herramienta. Las diferencias entre ambas condiciones fueron testeadas a partir de un análisis multivariado de dos factores, el cual reflejó un efecto principal significativo en el uso del computador portátil (Wilks's Lambda: .10; $F(7,32)=40.04 ; p<.01$ ). A partir de un análisis univariado también se encontró que el uso de la pizarra y cuadernos (herramientas consideradas de uso "tradicional") fue significativamente diferente en las dos condiciones ( $F(1,38)=9,62 ; p$ $=.04) ;(\mathrm{F}(1,38)=52,37 ; \mathrm{p}<.01)$ respectivamente.

También se encontró a partir de un análisis multivariado que la interacción entre el uso del portátil y las áreas de alemán, inglés y matemáticas, tiende a ser significativo [Wilks's Lambda = .53; $F(14,64)=1,69$; $p=.08]$. Particularmente se observó que en matemáticas decrece el uso de herramientas tradicionales dentro del aula, mientras que en las otras áreas no es tan clara la diferencia (Schaumburg, 2001).

La mayoría de las investigaciones revisadas muestran como este tipo de metodología ha sido usada mayoritariamente con población universitaria, donde sus estilos de aprendizaje y motivación para aprender están claramente definidos. Lo anterior origina que se genere en su mayoría un trabajo en forma individual más que grupal, y que por tanto, no se desarrolle un aprendizaje colaborativo. Roschelle et al (2001) afirma que no todas las formas o aplicaciones tecnológicas puestas en práctica en el aula, son efectivas para que 
los estudiantes alcancen logros en lo que aprenden y cómo lo zprenden. A continuación se presenta un avance en el uso de CSCL en la modalidad de computadores inalámbricos.

\section{c) Aprendizaje colaborativo soportado por computadores inalámbricos (ADP)}

Las ADP son consideradas como una herramienta común en el trabajo colaborativo (CSCW) pero que pueden llegar a ser herramientas innovadoras en el ámbito de la educación en aplicaciones de recolección y/o manipulación de datos, escritura, visualización, simulaciones etc (Topp \& Clark, 2003). Vahey \& Crawford (2003) afirman que los computadores portátiles inalámbricos han surgido como una herramienta que permite una mayor accesibilidad a todos los estudiantes a un bajo costo, y que se presenta como una herramienta que permite se alcancen logros positivos tanto en la enseñanza como en el aprendizaje.

Tempranas evaluaciones realizadas por Palm Education Pioners (PEP) en Estados Unidos evidencia que $90 \%$ de los profesores encuestados que trabajan con dicha herramienta afirman que contribuye positivamente al aprendizaje de los estudiantes, y el $84 \%$ de ellos concluye que el uso de ésta mejora la calidad de las actividades que como profesores implementaron con sus estudiantes (Vahey \& Crawford, 2003).

\section{Características de la tecnología en sí}

Los computadores inalámbricos traen consigo cuatro características que le son inherentes: portabilidad, movilidad, accesibilidad, y adaptabilidad (Pownell \& Bailey, 2000). La posibilidad de acceder a ella en cualquier tiempo y lugar, amplía el campo de acción del proceso de enseñanza aprendizaje. Por ejemplo puede ser usada dentro del aula para resolver una tarea de ciencias en conjunto con sus pares y también pueden utilizarla fuera del aula resolviendo una experiencia de campo relacionada con esta misma área. Esta facilidad brinda la posibilidad al estudiante de experimentar en contextos más cercanos a la realidad, generando en ellos una mayor autonomía y responsabilidad en su proceso de aprendizaje. A la par de lo anterior, las ADP facilitan el trabajo en grupos pequeños como un soporte entre compañeros, así los estudiantes pueden formar sus ideas, centrar su atención en la tarea y profundizar en conjunto en la solución de la misma (Vahey \& Crawford, 2003).

Dicha tecnología permite complementar la actividad propuesta con el acceso que puede tener a internet desde un computador fijo, "los estudiantes pueden acceder a la web, bajar páginas relevantes a su ADP para futuros análisis" (Vahey \& Crawford, 2003, p.8). Aunque esta característica se perfila como relevante para un trabajo más profundo, los profesores han reportado que esta opción puede transformarse en un estímulo disruptivo en el aula (Tatar et al, 2003).

En general existen dos formas de asignación de esta herramienta ya sea de uso personal en cuyo caso cada estudiante tendría su propio ADP, o el uso de los ADP en áreas y actividades específicas. Con la primera modalidad se genera un problema más de tipo económico pues implica un alto costo para la organización escolar, por lo que generalmente se usa la segunda modalidad. Actualmente en el contexto chileno se han iniciado experiencias piloto haciendo uso de la segunda modalidad y aplicándola en las áreas de castellano, biología, química, física y matemáticas (Rosas, Correa, Flores, Grau, Lagos, López, Troncoso, \& Nussbaum, 2003). Éstas tienen poco tiempo de aplicación en el contexto lo que hace que aún no exista sistematización de datos. 


\section{Beneficios y desventajas del uso de ADP en el contexto escolar}

Tatar et al (2003) realizó una evaluación a gran escala durante un año con profesores y estudiantes que hicieron uso de dicha tecnología. Encontraron que los profesores reportan un incremento en los aprendizajes de sus estudiantes, en las interacciones colaborativas, y en la autonomía del estudiante sobre las actividades propuestas. En cuanto a las desventajas reportadas, éstas guardan relación con el uso inapropiado de la información o el contenido que pueden bajar los estudiantes en un momento determinado, o problemas de la tecnología específicamente en el daño del equipo.

En la literatura revisada los hallazgos reportan que los estudiantes mantienen la atención sobre la tarea cuando trabajan con las ADP y que el rol del profesor juega un papel importante en este trabajo. Observaciones realizadas en un estudio con grupos de 4 y 5 básico, muestran como los estudiantes raramente se distraen de la tarea que están llevando a cabo y que por el contrario, presentan una gran flexibilidad dentro del grupo en la forma como organizan su trabajo. Por otro lado los profesores en este tipo de CSCL monitorean en forma constante el trabajo de todos los grupos propiciándoles ayuda a todos sus estudiantes, llegando a ser concientes de que sus intervenciones fueron efectivas para el uso de la herramienta. Tanto profesores como estudiantes reconocen que existe un mejor trabajo con las ADP cuando el profesor se encuentra cerca (Yarnall, Penuel, Ravitz, Murray \& Means, 2001).

Un segundo estudio realizado por este mismo equipo de investigadores, pero esta vez con 41 estudiantes de sexto grado, de las áreas del lenguaje del arte y estudios sociales, encontraron que en este nivel intermedio la dinámica social difiere notoriamente de la que se muestra desde un principio en el nivel básico. En éste se presentan más conductas disruptivas, competitivas o detractoras con la actividad propuesta, pero con el correr del tiempo, en la actividad, los estudiantes van cambiando su actitud hacia aquellas que le permiten alcanzar logros colaborativamente. Tanto profesores como estudiantes coinciden en que el lenguaje que se presenta en la herramienta puede llegar a ser un factor que dificulte el trabajo en los grupos CSCL.

Roschelle et al (2001) reporta que los beneficios que se obtienen al trabajar con ADP abarca un compromiso activo de los estudiantes, una frecuente interacción y retroalimentación entre ellos, que genera dentro del grupo una conexión directa con el mundo real. Estas características no son exclusivas de las actividades planteadas con ADP, pero lo relevante es que al trabajar con ellas se encuentra que los estudiantes presentan un incremento mayor en estas habilidades que el obtenido cuando se trabaja sin ellas.

Danesh, Inkpen, Lau, Shu y Booth (2001) construyeron una aplicación educacional (Geney) que le permitiría a los niños aprender conceptos de genética, resolver problemas en un contexto exploratorio y, desarrollar habilidades de colaboración para alcanzar un logro común. Su diseño, basado en el principio de centrado en el usuario (en el estudiante en este caso), pretendía favorecer sus relaciones de colaboración. Los resultados obtenidos resaltan la riqueza de las interacciones sociales que se generan a partir de su aplicación, así como también la extensión de las relaciones colaborativas hacia otros grupos cercanos y en consecuencia, la expansión de sus redes sociales.

Mandryk, Inkpen, Bilezikjian, Klemmer \& Landay (2001) usaron la misma aplicación educativa pero con una variante: múltiples aparatos interconectados y con una nueva característica en Geney, la condición what-if que provee información que el niño puede usar para tomar decisiones en conjunto con sus pares. 
Los resultados obtenidos muestran un incremento en las interacciones cara a cara y en la síntesis de información. Una de las limitaciones de dichos estudios como lo afirman los autores, es justamente la naturaleza informal de la recolección de los datos que les permiten hacer las aseveraciones anteriores. En este sentido se hace necesario implementar técnicas de recolección de datos que sin alejarse del contexto, puedan ser generalizados con mayor fundamento empírico.

En general se encontró que el aprendizaje colaborativo soportado con ADP se ha desarrollado en su mayoría en niveles escolares medios e intermedios, así como en los universitarios. Asimismo las áreas en donde más se ha llevado a cabo su aplicación ha sido en las matemáticas o la física, aunque actualmente se ha iniciado el trabajo con ADP en áreas como los estudios sociales, el lenguaje y el arte (Roschelle et al, 2001).

Estos últimos autores afirman que en comparación con el uso de lápiz y papel que soporta notaciones aisladas y estáticas en las matemáticas, el uso de computadores permite el aprendizaje de los mismos conceptos de manera más dinámica. Por ejemplo con el proyecto Simcalc los estudiantes han incrementado sus habilidades en relación al aprendizaje de conceptos de proporción, velocidad entre otros, por medio de la manipulación y simulación de movimientos en gráficas. En estos escenarios, Roschelle, Vahey, Tatar, Kaput \& Hegedus (2003) afirman que los discursos emitidos guardan relación directa con el contenido matemático y que en consecuencia, las conductas disruptivas se observan poco.

\section{3) Unidad de análisis: las interacciones y su evaluación}

Algunas investigaciones sugieren que los procesos de aprendizaje son más rápidos cuando el estudiante tiene mayor posibilidad de compartir y discutir sus ideas, y de recibir retroalimentación inmediata de ellas por sus compañeros o profesores. La posibilidad de recibir comentarios que le permitan evaluar sus ideas les facilita a los estudiantes el poder trabajar por periodos de tiempo en forma individual y grupal (Roschelle et al, 2001). Mandryk et al (2001) concluyen en su estudio que la riqueza de las interacciones cara a cara ayudan a los niños a sintetizar información y a crear una dinámica de colaboración y compromiso con el aprendizaje.

Algunas investigaciones apuntan a que la clave del éxito de las interacciones esta en el rol que el profesor juega en la dinámica que se origina dentro del aula. Roschelle \& Pea (2002) afirman que el profesor debe atender mayoritariamente la ejecución del grupo y no la individual. Por tanto su función se ve enmarcada por la interpretación continua de las ejecuciones que ocurren al interior del grupo, y de propiciar la ayuda necesaria a los estudiantes que les sirva como puente en la construcción de su conocimiento. En este sentido el profesor se constituye en un conductor del aprendizaje que tiene la responsabilidad de elegir las actividades curriculares que se implementarán, y además, de facilitar el camino a los estudiantes para que alcancen los logros deseados.

En general se encontró que el estudio de las interacciones ha empezado a ocupar un lugar importante en el estudio de ambientes de aprendizaje colaborativo soportado con computadores (Inaba, Supnithi, Ikeda, Mizoguchi \& Toyoda, 2000; Martínez, Dimitriadis \& De la Fuente, 2002). Por ejemplo Barros, Mizoguchi \& Verdejo (2001) construyeron una plataforma (DEGREE) que permite el análisis de la colaboración que se da en los grupos a partir del análisis de las interacciones. Este método de análisis permite el estudio de las diferentes etapas que ocurren en una discusión argumentativa. Los resultados de su aplicación permiten identificar en las interacciones el grado de divergencia, balance o fluencia en la 
conversación y en consecuencia, la información en relación a como los estudiantes trabajan para resolver la tarea.

Martínez, Osuna, Becerril, Dimitriadis, De la Fuente, Barrio \& Blasco (2001) afirman que el estudio de las interacciones puede proporcionar información en relación a los procesos que se llevan a cabo en CSCL, el cual debe integrar datos cuantitativos y cualitativos haciendo uso de métodos etnográficos y analíticos. Las fuentes etnográficas más usadas son las observaciones, cuestionarios e intervenciones, capaces de capturar las percepciones de los participantes. Mientras que los datos cuantitativos generalmente son usados para contar el número de veces o la ocurrencia de acciones o eventos los que se relacionan con los datos obtenidos cualitativamente (Martínez, Dimitriadis, Rubia, Gómez \& De la Fuente, 2003).

De esta manera se han creado sistemas que potencian el desarrollo de habilidades en los estudiantes y a la vez, recogen información de los tipos de interacciones que pueden producirse. Por ejemplo, PENCACOLAS (PEN Computer Aided Collaborative System) es un sistema usado para enseñar técnicas de escritura de manera colaborativa en la Facultad de Educación de la Universidad de Valladolid. Este sistema plantea un problema que permite de manera recursiva el uso de diferentes fases como por ejemplo lluvia de ideas, planeación, escritura y revisión. Asimismo da la posibilidad de volver sobre estas fases, pues almacena todo el trabajo que se ha llevado a cabo hasta ese momento.

PENCACOLAS integra la recolección de datos cuanti y cualitativos, considerando tanto los eventos o mensajes producidos como las acciones ejecutadas. Los resultados de su aplicación indican la existencia de dos categorías de interacciones: las activas y las potenciales. Éstas indican la intervención de un miembro en el trabajo de otro miembro del grupo y la presencia de una posible interacción, respectivamente. El tiempo es una dimensión reconocida como importante de la colaboración, por lo cual se considera dentro de la información contextual (Martínez et al, 2001).

Es relevante destacar que dentro de este sistema se consideran tres niveles de tratamiento de los eventos: a) la forma de almacenaje de las participaciones (p.e. tiempo, sesión, autor, etc.), b) el análisis de la participación de cada miembro del grupo y de las relaciones que se dan dentro del grupo y, c) el tratamiento semántico, es decir, la definición de la categoría de interacción. Aunque este sistema está en proceso de perfeccionamiento, permite obtener el tiempo que tarda el grupo en una fase determinada y conductas inusuales que se puedan presentar dentro de la interacción, en orden a detectar cuales son los patrones de interacción que mejor provee procesos de aprendizaje (Martínez et al, 2001).

En esta misma línea se han construido plataformas que permite evaluar el contenido de las interacciones así como las actitudes hacia la colaboración. En relación al contenido de las interacciones, Martínez et al (2002) plantea una forma de evaluar las interacciones capturando los eventos y procesándolos para modelar el estado de la interacción. A partir de un programa que delinea en términos computacionales el contenido de las interacciones para almacenarlas posteriormente en un fichero, intenta evaluarlas de manera genérica y no con taxonomías que pertenezcan a una situación determinada. Esta se constituye en una línea de investigación incipiente y por tanto, los resultados que se han alcanzado hasta el momento hacen parte de la perfección del modelo.

Otros estudios han combinado fuentes de datos y métodos de análisis cuanti y cualitativos que han permitido elevar la confiabilidad de los resultados que se dan a partir de la colaboración. Por ejemplo LAO 
(Laboratorio de Arquitectura de Ordenadores) es un proyecto usado como una plataforma para la investigación educacional que permite evaluar tanto el impacto del diseño pedagógico de la herramienta como las actitudes colaborativas generadas en el trabajo entre pares. Éste se utilizó en una muestra de estudiantes universitarios de la Escuela de Ingeniería de Telecomunicaciones de la Universidad de Valladolid, los cuales organizados por parejas trabajaron durante 15 semanas para resolver una tarea que consistía en diseñar y evaluar sistemas computacionales.

Diferentes técnicas de análisis como observaciones formales, focus group, cuestionarios, entre otros, se usaron para obtener la información. Los resultados obtenidos después de trabajar en el proyecto reportan que: a) se presenta un incremento notable en las habilidades como discusión (48\%), resolver dudas (11\%), compartir información (53\%) y crear un producto común (82\%), b) un $40.9 \%$ de estudiantes consideran que la colaboración se da solo con amigos y, c) aunque existe una percepción positiva por parte de los estudiantes en relación a sus propias actitudes colaborativas en el grupo, éstas se presentaron por razones practicas más no porque tuvieran como objetivo la formación de su propio conocimiento (Martínez et al, 2003).

\section{FUTURAS DIRECCIONES Y CONCLUSIONES}

La revisión de la iteratura encontrada hasta el momento sobre CSCL inalámbricos nos permite aventurarnos a plantear algunas futuras direcciones a investigar. A continuación se presenta un punteo de los posibles campos de acción que pueden permitirnos alcanzar algunos hallazgos -esperamos- que enriquezcan el marco conceptual de este nuevo paradigma.

Investigación en niveles escolares: La mayoría de las investigaciones realizadas apuntan al estudio de esta modalidad de trabajo en espacios universitarios. Se requiere entonces, investigaciones que tomen un espectro más amplio de la población escolar (básica-media). Por ser un campo en vías de expansión los estudios llevados a cabo con estudiantes de media, presentan hallazgos contradictorios en relación a las actitudes colaborativas que se desarrollan, no así en los alcances que puede llegar a tener a nivel cognitivo. Lo anterior implica la necesidad de poder, a partir de variados estudios con población adolescente, determinar las condiciones aptas para la potenciación de actitudes positivas hacia la colaboración.

Calidad de la interacción: La literatura revisada expone que la unidad de análisis del CSCL es la interacción. Ésta se constituye en la unidad que permite evaluar la calidad de la colaboración, y en el fondo la calidad del proceso de enseñanza aprendizaje que se está llevando a cabo en los trabajos CSCL. En este sentido, el estudio de las interacciones se constituye en el elemento clave para entender el proceso de aprendizaje que se genera, intergrupal e intraindividual.

Esta revisión empírica también revela la importancia de centrar la atención en la estructura de la tarea, aspecto que se encuentra estrechamente relacionado con la interacción. Una tarea estructurada a partir de CSCL fijos o con ipaq genera procesos de aprendizaje diferentes en los estudiantes. La manera en que se estructure la tarea genera o propicia la aparición de diferentes tipos de interacciones, por tanto, los resultados obtenidos de tareas estructuradas teniendo como centro el estudiante serán diferentes a aquellas centradas en la actividad como fuente de desarrollo de aprendizajes. Estructura de tareas fijas, estáticas, que no permiten al estudiante interactuar en forma dinámica, generan resultados cognitivos y sociales diferentes, que aquellas que se estructuran en forma contraria. En general muy pocas investigaciones se 
encontraron apuntando al estudio de las interacciones en CSCL con ADP lo que se constituye en un terreno fértil de investigación.

Junto con el estudio de las interacciones en los estudiantes dentro de cada grupo, también se destaca el rol que debe desempeñar el profesor dentro de la dinámica. En las investigaciones revisadas el rol del profesor es pasado por alto, y se centran mayormente en los estudiantes. En este sentido es interesante apuntar a dilucidar los roles de todos los actores que participan en la dinámica establecida, con el fin de llegar a plantear cual es el clima escolar adecuado, los requerimientos que se necesitan para implementar este método instruccional dentro del aula.

Metodología para evaluar el aprendizaje colaborativo: Actualmente, las investigaciones revisadas apuntan al estudio del CSCL a partir de metodologías cuantitativas y cualitativas. En la modalidad inalámbrica (ADP) las investigaciones a nivel cualitativo son más recientes. Éstas tienen que ver con la creación de plataformas o estructuras de tareas que permitan evaluar el contenido del área, pero a su vez las interacciones que se generen dentro de esta dinámica. Es lo que Martínez et al (2002) ha denominado evaluación formativa del CSCL. La combinación de dichas metodologías se constituye en un aspecto clave al momento de evaluar el trabajo con ADP, en especial la metodología cualitativa que ha estado relegada por mucho tiempo y de la cual podemos obtener información que enriquece el trabajo con esta tecnología a partir de las percepciones que tienen los diferentes actores sociales que llevan trabajando con este tipo de diseño instruccional.

\section{CONCLUSIONES}

Los resultados que se han obtenido a partir de las investigaciones muestran los hallazgos positivos que se pueden obtener al trabajar con este tipo de tecnología dentro del aula. Asimismo nos indican la riqueza en las interacciones y la posibilidad que tiene el estudiante de construir conocimiento en forma activa, siendo conciente de su propio proceso de aprendizaje. El uso de dicha tecnología también ha indicado los aportes que genera a la estructuración del currículo y de la organización escolar.

Si partimos entonces de los beneficios que se puede dbtener al trabajar con las ADP, entonces se hace necesario seguir investigando en relación al tipo de interacciones que surgen al trabajar con dicha herramienta. Su estudio más detallado nos permitirá entender más la dinámica que se genera al aprender colaborativamente y cuál es el apoyo o sostén que le puede brindar a la evaluación formativa por parte del docente.

Las investigaciones que se realizan a partir de este nuevo paradigma abren una puerta en el estudio del marco teórico que lo sustenta. Se hace necesario que se lleven a cabo investigaciones que apunten a la fundamentación de dicho paradigma, reconocer bajo que perspectiva se hace más efectivo su estudio, cuales son los supuestos que se encuentran en su base, y finalmente cual es el cimiento que sostiene este método instruccional y que lo hace relevante en el contexto escolar.

\section{BIBLIOGRAFÍA}

BARRO S, Beatriz, MizoguCHI, Riichiro. \& VerdeJo, Felisa."A platform for collaboration analysis in CSCL. An ontological approach" .Proceedings Artificial Intelligence in Education AllED'2001. [En red] http://sensei.ieec.uned.es (2001). 
COLE, Michael. Psicología cultural: una disciplina del pasado y del futuro [traducción de Tomás del Amo] Madrid: Morata (1999).

DANESH, Arman, INKPEN, Kori, LAU, Felix, SHU, Keith \& BOOTH, Kellogg. Geney TM: designing a Collaborative Activity for the palm TM Handheld Computer. [En red]

www.edgelab.sfu.ca/publications/chi2001_geney.pdf (2001)

Dillenbourg, Pierre. Collaborative Learning. Cognitive and Computational Approaches. New York: Pergamon Earli (1999).

GIFFORD, Bernard. \& ENYEDY, Noel D. Activity centered design: Towards a theoretical framework for CSCL. Proceedings of the Third International Conference on Computer Support for Collaborative Learning. [En red] www.gseis.ucla.edu/faculty/enyedy/pubs/ Gifford\&Enyedy_CSCL2000.pdf. (1999).

INABA, Akiko, SUPNITHI, Thepchai, IKEDA, Mitsuru, MizOGUCHI, Riichiro \& TOYODA, Jun'ichi. ¿How Can We Form Effective Collaborative Learning Groups? [En red] http://www.ai.sanken.osakau.ac.jp/indexe.html. (2000).

LÊ, Thao. Collaborative to learn and learn to collaborate. Australian Computer Society, Inc. Paper presented at the Seventh World Conference on Computers in Education, Copenhagen, July 29- August 3. (2002).

LEA, Martin, RogERS, Paul \& POSTMES, Tom. SIDE-VIEW: Evaluation of a system to develop team players and improve productivity in Internet collaborative learning groups. British Journal of Educational Technology, 33 (1), 2002, 53-63.

LUCERO, Maria. Entre el trabajo colaborativo y el aprendizaje colaborativo. Revista Iberoamericana de Educación (ISSN: 1681-5653) [En red:] http:// www. Capmpus.oei.org/revista/de los lectores/ 528/Lucero. pdf (2003)

MANDRYK, Regan, INKPEN, Kori, BILEZIKJISN, Mark, KLEMMER, Scott \& LANDAY, James Exploring a New Interaction Paradigm for Collaboration on Handheld Computers.[En red:] http://guir.berkeley.edu/projects/geney/GeneyTechReport.pdf.(2002)

MANDRYK, Regan, INKPEN, Kori, BILEZIKJISN, Mark, KLEMMER, Scott \& LANDAY, James. Supporting Children's Collaboration Across Handheld Computers. [En red:]

http://www.edgelab.sfu.ca/publications/chi2001_handheld.pdf. (2001)

MartíneZ, Alejandra., DimitriAdIS, Yannis \& DE LA Fuente, Pablo. Aportes al análisis de interacciones para la evaluación formativa en CSCL. Presentado para su revisión al IE. VIGO: España. [En red:] pireo.tel.uva.es/gsic/usuarios/all/../../papers/paper26.pdf . Nov. 2002.

MARTíneZ, Alejandra., Dimitriadis, Yannis., RUBiA, Bartolomé, GómeZ, Eduardo \& DE LA FUENTE, Pablo. Combining qualitative evaluation and social network analyses for the study of classroom social interactions. Computer and Education, 41 (4), 2003, 353-368.

MARTínEZ, Alejandra, DIMITRIADIS, Yannis, RuBIA, Bartolomé, GómEZ, Eduardo, GARACHÓN, Inmaculada \& MARCOS, José. Studying social aspects of computer-supported collaboration with a mixed evaluation 
approach. In Proceedings of Computer Support for Collaborative Learning Conference, CSCL 2002, Boulder, CO, USA. (2002).

Martínez, Alejandra, Osuna, Cesar, BeCERRIL, Ana., Dimitriadis, Yannis, Fuente, Pablo DE LA, BarRio, José \& BLASCO, Teresa. Modeling interactions with a conceptual framework. [En red] http://collide.informatik.uni-duisburg.de/ecai-2000/W26_Martinez.pdf (2000).

MEANS, Barbara. New SRI International Study Handheld Computers Can Increase Learning in K-12 Classrooms. [En red.] http://www.sri.com/news/releases/11-11-02.html. November 11, 2002.

MUHLENBROCK, Martin. A system for Analyzing Collaborative problem solving [En red] http://citeseer.nj.nec.com/410548.html (1999).

POWNELL, David \& BAILEY, Gerald. Handheld Computing for Educational Leaders: A Tool for Organizing or Empowerment. Leading \& Learning with Technology. (May 2000).

RoGofF, Bárbara. Aprendices del pensamiento El desarrollo cognitivo en el contexto social. España: Paidos (1993).

Rosas, Ricardo, Correa, Mónica, Flores, Patricia, Grau, Valesca., LAGOS, Francisca, LóPeZ, Verónica., TRONCOSO, Ana María \& NUSSBAUM, Miguel. Diseño y desarrollo de un sistema generador de pruebas de evaluación de aprendizaje escolar a través de computadores portátiles inalámbricos. Revista Pensamiento Educativo, Vol. 33, (2003), 128-159.

ROSCHELLE, Jeremy \& PEA, Roy. A Walk on the WILD side: How wireless handhelds may change computer supported collaborative learning. [En red:] http://newmedia.colorado.edu/cscl/79.pdf (2002).

Roschelle, Jeremy, PeA, Roy, HoAdley, Christopher, Gordin, Douglas \& MEANS, Barbara. Changing How and What Children learn in School with Computer-Based Technologies. The future of Children, 10 (2), Los altos, CA: Packard Foundation, (2001), 76-101.

Roschelle, Jeremy, VAhey, Phil., TAtaR, Deborah., KaPut, Jim \& Hegedus, Stephen. Five key considerations for networking in a handhelds-based mathematics classroom. In N.A. Pateman \& B.J. Dougherty \& J.T.Zilliox (Eds.). Procedings of the 2003 Joint Meeting of PME and PMENA VolHonolulu, Hawaii: University of Hawaii, 4, (2003), 71-78.

SCHAUMBURG, Heike. The impact of mobile computers in the classroom- Results from an going video study.[En red] http://usight.concord.org/documents/electronic_guidebook.pdf. (2001).

TATAR, Deborah, Roschelle, Jeremy, VAHEY, Phil \& PenUel, William. Handhelds: Go to Scholl: Lessons Learned. Published by the IEEE Computer Society, (2003)

TOPP, Neal \& CLARK, Paul. Handhelds: Important Technology for Classrooms and Educators. [En red] http:// unomaha.edu/handhelds/handhelds.doc. (2002)

TORGRUDE, Marcia. Why Handhelds in the Classroom [En red] http://handheld.tie.net/basics/Why Handhelds (2001)

VAHEY, Phil \& CRAWFORD, Valerie. Learning With Handhelds: Findings From Classroom Research. [En red] www.intel.com/education/handhelds/SRI.pdf. (2003), 1-13. 
VEeRman, Arja. Collaborative learning through electronic discussion. [En red] http://collide.informatik.uniduisburg.de/ecai-2000/W26_Veerman.pdf (2000)

VeERMAN, Arja, ANDRIESSEN, Jerry \& KANSELAAR, Gellof. Collaborative Learning through Computer-Mediated Argumentation. Computer Support for collaborative learning, 640-651. [En red] citeseer.nj.nec.com/veerman99collaborative.html - 20k (1999).

VIGOTSKY, Lev. Interacción entre aprendizaje y desarrollo. En M. Cole, V. Steiner, S. Scribner \& E. Souberman. (Eds.), El desarrollo de los procesos psicológicos superiores. Barcelona: Crítica,(1996).

YARNALL, Louise, PenUel, William, RAVITZ, Jason, MuRRAY, Grahame \& MeANS, Bárbara. Portable Assessment Authoring: Using Handheld Technology to Assess Collaborative Inquiry. Paper to be presented at Annual Meeting of the American Educational Research Association, Seattle, Washington. (April, 2001). 


\title{
Contactar
}

Revista lberoamericana de Educación

\author{
Principal OEI
}

